LA-UR-01-1400

Approved for public release; distribution is unlimited.

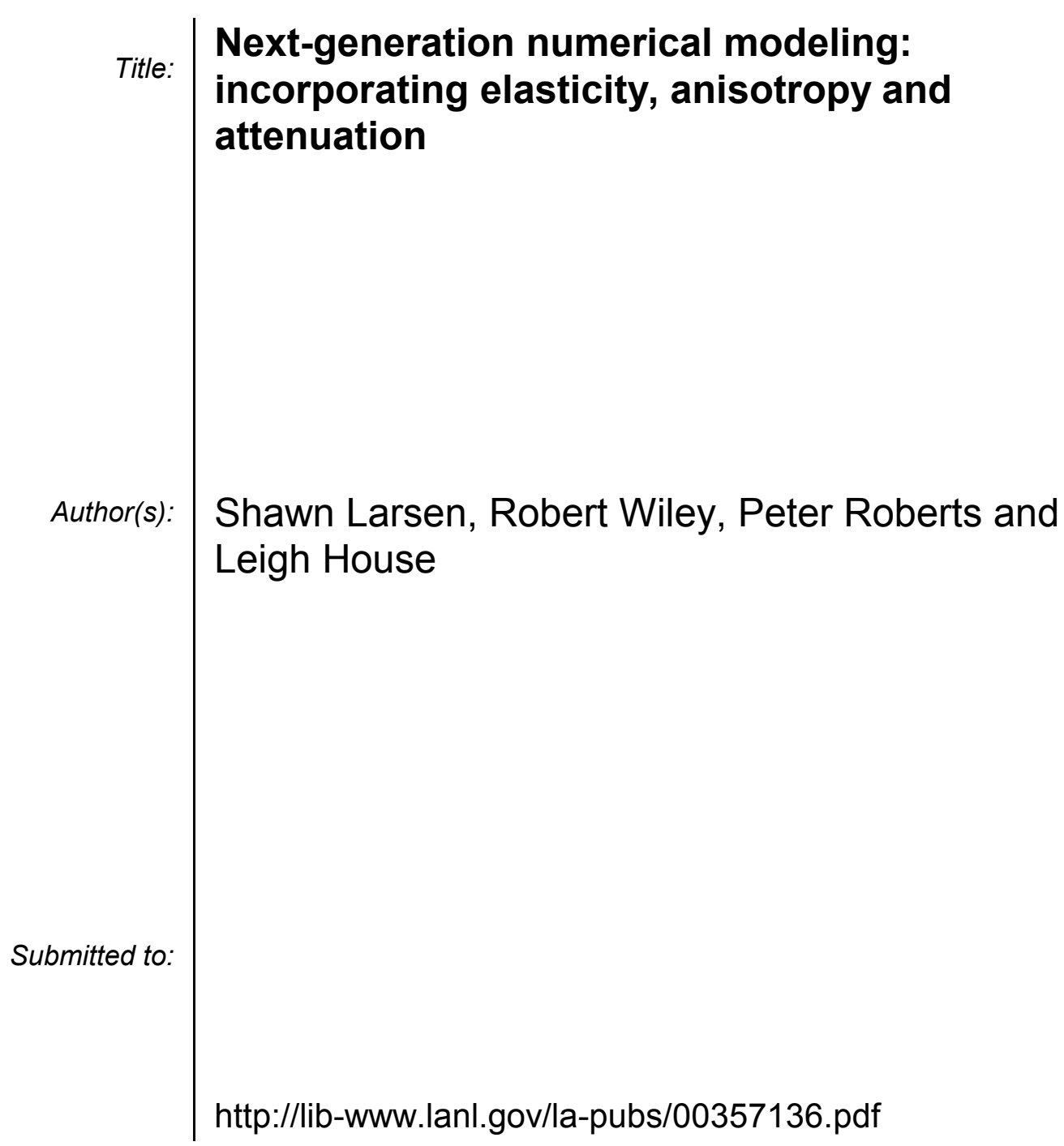

Los Alamos National Laboratory, an affirmative action/equal opportunity employer, is operated by the University of California for the U.S. Department of Energy under contract W-7405-ENG-36. By acceptance of this article, the publisher recognizes that the U.S. Government retains a nonexclusive, royaltyfree license to publish or reproduce the published form of this contribution, or to allow others to do so, for U.S. Government purposes. Los Alamos National Laboratory requests that the publisher identify this article as work performed under the auspices of the U.S. Department of Energy. Los Alamos National Laboratory strongly supports academic freedom and a researcher's right to publish; as an institution, however, the Laboratory does not endorse the viewpoint of a publication or guarantee its technical correctness. 


\section{Next-generation numerical modeling: incorporating elasticity, anisotropy and attenuation}

Shawn Larsen*, Lawrence Livermore National Laboratory, Robert Wiley, University of Houston, Peter Roberts and Leigh House, Los Alamos National Laboratory

\section{Summary}

A new effort has been initiated between the Department of Energy (DOE) and the Society of Exploration Geophysicists (SEG) to investigate what features the next generation of numerical seismic models should contain that will best address current technical problems encountered during exploration in increasingly complex geologies. This collaborative work is focused on designing and building these new models, generating synthetic seismic data through simulated surveys of various geometries, and using these data to test and validate new and improved seismic imaging algorithms. The new models will be both 2- and 3-dimensional and will include complex velocity structures as well as anisotropy and attenuation. Considerable attention is being focused on multicomponent acoustic and elastic effects because it is now widely recognized that converted phases could play a vital role in improving the quality of seismic images. An existing, validated 3-D elastic modeling code is being used to generate the synthetic data. Preliminary elastic modeling results using this code are presented here along with a description of the proposed new models that will be built and tested.

\section{Introduction}

The U.S. oil and gas industry needs to obtain better seismic images of reservoirs to continue to explore and produce from ever more complex geologic structures. These reservoirs are difficult to image properly using conventional 3-D imaging methods because those methods do not adequately take in to account all of the wave phenomena that can occur in complex structures. Some structures produce mode conversions (elastic wave effects) that are not imaged properly and that produce misleading artifacts (e.g., Ogilvie and Purnell, 1996, Kessinger and Ramaswamy, 1996). In other cases, sharp geological discontinuities bend and distort seismic waves and cause severe multipathing. In the most complex cases both elastic and multipathing effects combine to make accurate imaging extremely challenging. In addition to producing artifacts, however, elastic waves provide valuable additional information. To better exploit that information, the exploration industry is collecting multi-component seismic data. Multicomponent data can provide better imaging in some situations (such as shallow gas columns), and can provide rock parameter information that is essential for reservoir modeling. Processing and interpreting multi-component data adds to the already heavy computational burden of conventional 3-D seismic data, and requires a much better understanding of elastic wave propagation than what we now have. To overcome these challenges and to better exploit the opportunities provided by these new data, new research efforts must be aimed at increasing our understanding of 3-D elastic wave propagation effects in complex geological structures, including the effects of viscoelastic attenuation and anisotropy.

A collaborative next generation numerical modeling effort has been initiated between industry, academia, SEG and two DOE national laboratories. A major task of this effort is to develop new elastic models that will provide standard synthetic data for testing new imaging technologies. A 3-D, finite difference, elastic modeling algorithm has been developed that takes advantage of the speed and power of current high-performance computer technology. The modeling code, called E3D, is able to generate multi-component synthetic seismic data from 3-D elastic models, is faster than a previously developed acoustic modeling algorithm and has been widely tested and accepted by industry. To provide guidance for designing the new models, the SEG Research Committee has formed a Modeling Subcommittee which receives and assesses design suggestions from seismologists and reservoir engineers. Design features for several new models already have been proposed and are described below.

Elastic and other nontraditional techniques are significantly more numerically intensive than acoustic modeling. For example, a 3-D elastic model representing a typical off-shore exploration environment requires approximately 250 times the computational resources as a similar acoustic model. Models incorporating viscoelastic attenuation, anisotropy, and topography are even more computationally intensive. Hence, there is a need to apply advanced computational technologies that utilize high performance computing and massively parallel processing. This is important because the oil and gas industry is requiring greater use of sophisticated modeling techniques to find and develop hydrocarbon resources from increasingly subtle and complex geologic settings.

\section{Method}

Next Generation Model Designs

The Modeling Subcommittee has been partially formed and proposals for what features the new models should contain are being considered. Current plans are to develop both 2-D and 3-D elastic models to use as input to the E3D code described below. The 2-D models will simulate anisotropic problems 


\section{Next-generation numerical modeling}

that are typically encountered in complex geology. The 3-D model will be based on an existing Gulf of Mexico (GOM) subsalt target that has been well characterized. One or more standard data sets will be generated with known parameters that can be used to test and develop new technologies for imaging and understanding anisotropic and 3-D elastic effects. One data acquisition scenario is to position an array of 3component receivers just below the water/sediment interface in the model to provide a realistic 3-component response. Also, a hydrophone will be placed just above the water/ sediment interface to simulate the pressure response. This scheme will produce the full 4-component data acquisition for a typical ocean bottom cable survey. The GOM model that will be used for this simulation is based on an actual offshore field. To further extend the applicability of the synthetic data, input from reservoir engineers is being used to model the reservoir as accurately as possible. This will include features that incorporate reservoir scale properties.

\section{Elastic 3D Modeling}

A robust 3-D finite-difference wave propagation code capable of realistically simulating elastic waves in large 3-D geologic models has been developed at Lawrence Livermore National Laboratory (Larsen and Grieger, 1998). This code, known as E3D, is being used for a number of diverse geophysical and other research projects. These projects include seismic hazard evaluation, nuclear nonproliferation, underground structure detection, geodetic analysis, hydroacoustics research, tectonic studies, optics, medical imaging, and oil exploration. The code was developed by leveraging the technical and computational needs of several scientific projects into a single wave propagation framework (Figure 1).

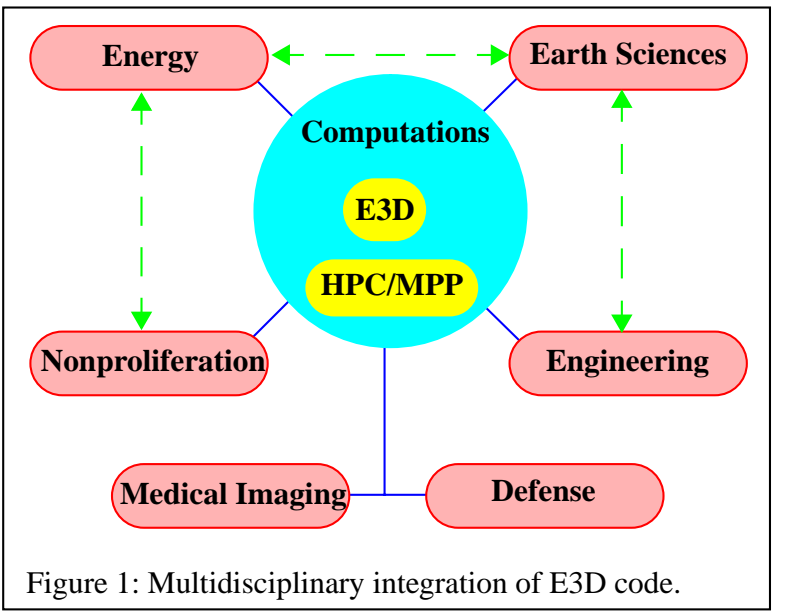

E3D contains physics-based and computational enhancements. Physics-based enhancements allow more accurate simulations of wave propagation and include full 3-D elastic and viscoelastic modeling (Robertsson et al., 1994) and the capability to incorporate the effects of surface topography on seismic energy (Schultz, 1997). Anisotropic modeling capabilities are being incorporated (e.g., Mora, 1989, Carcion, 1996). Computational enhancements make it possible to simulate larger problems more efficiently and include lowlevel optimization, propagating and variable density grids (e.g., Berger and Colella, 1989, McLaughlin and Day, 1994), hybridization, and parallelization. E3D runs on a variety of platforms, including desktop workstations, Linux clusters, vector processors, and massively parallel processors (MPP's). The code is heavily optimized. Performances of up to $57 \%$ peak processor speed have been observed.

E3D is based on the elastodynamic formulation of the full wave equation on a staggered grid (e.g., Madariaga, 1976; Virieux, 1986; Levander, 1988). Grid velocities and stress tensor components are solved by an explicit finite-difference scheme, whereby each variable is staggered by $1 / 2$ grid point from the other variables (except that all normal stresses are computed on the same grid). A 4th-order spatial stencil is applied over each variable and a 2nd-order explicit method is used to propagate the simulation with time. Body force functions and/or seismic moment rates are used as source terms to drive the velocities and stresses.

The staggered grid implementation is beneficial because the spatial differencing stencils are centered around each variable, which minimizes the computational burden for a given level of accuracy. In addition, the 2nd-order time stepping technique allows all equations to be updated in place. This reduces the memory requirement. For 3-D purely elastic simulations, 12 state variables are required at each grid point $(3$ medium parameters, 3 components of velocity, and the 6 independent members of the symmetric stress tensor); 2-D problem require 8 variables at each grid point.

Three-mechanisms are used to minimize artificial reflections at the side and bottom boundaries of the numerical grid: 1) absorbing boundary conditions using paraxial extrapolation (Clayton and Enquist, 1977); 2) sponge boundary conditions using an amplitude reduction coefficient applied over a swath of grid nodes parallel to the boundary (Cerjan et al., 1984); 3) attenuation boundary conditions using a low $Q$ factor applied at nodes near the grid boundary. The free-surface is modeled as either a horizontal plane (e.g., Graves, 1996) or as a boundary of topographic relief using a density extinguishing approach (Schultz, 1997).

E3D is implemented on several high performance computing architectures, massively parallel processors. A massively parallel machine typically has between 32 and 8192 nodes or CPU's, each operating quasi-independently on a subset of a larger problem. Extremely powerful massively parallel computers are being developed as part of the Department of Energy's ASCI initiative. In fact, E3D is about to be implemented on ASCI-White, the most powerful computer in 


\section{Next-generation numerical modeling}

the world. This machine has 8192 CPU's, 4.0 TeraBytes of internal memory, 190.0 TeraBytes of diskspace, and operates at a peak performance of 12.3 TeraFlops. For comparison, this machine is $10,000-20,000$ times more powerful than typical scientific desktop workstations. This magnitude of compute power is needed for 3-D simulations of large elastic models at sufficiently high frequency (e.g., $25 \mathrm{hz}$ full-physics seismic simulations over 3-D geologic models tens to hundreds of kilometers in dimension).

\section{Example}

The importance of simulating seismic energy through elastic and more complex geologic structures is illustrated by comparing a 3-D acoustic simulation through the SEG/EAGE subsalt model (e.g., House et al., 1996) with a simulation through an elastic representation of the same model (Figure 2). These models consist of a shallow water layer overlying sediments, with a salt body located about $2 \mathrm{~km}$ below the surface. Both simulations are performed using an explosive source in the water with central source frequency of $15 \mathrm{hz}$.

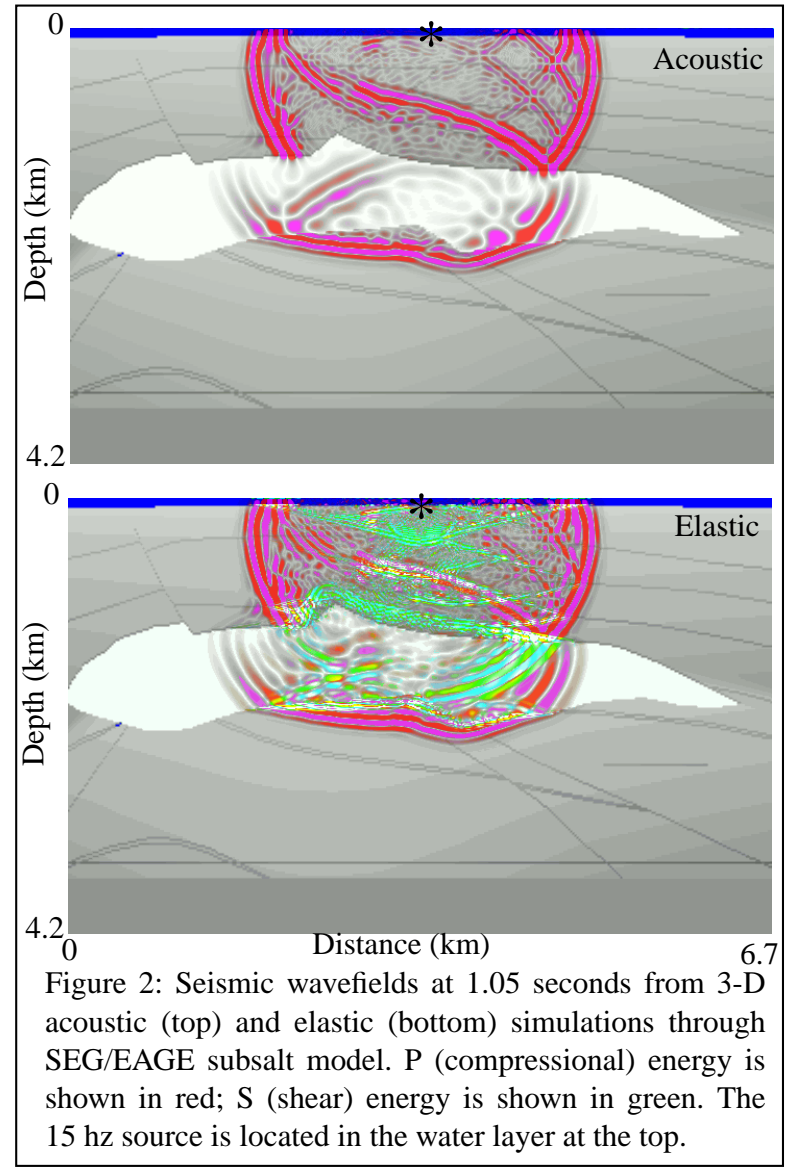

Snapshots of the seismic wavefield at 1.05 seconds indicate considerable differences between the acoustic (top) and elastic (bottom) simulations (Figure 2). P (compressional) energy is shown in red, $\mathrm{S}$ (shear) energy is shown in green. In the elastic simulation, mode-converted shear energy is generated at the water-sediment interface, and at the top and bottom boundaries of the salt body. In addition, mode-converted compressional energy is generated from the shear waves at the same interface boundaries. The increased complexity of the elastic simulation demonstrates the need to incorporate geologic complexity in the next generation of SEG numerical models. In this particular case, processing artifacts can be avoided through shear wave analysis, and the additional information provided by elastic modeling can be used to better image the underlying geology. This is particularly true when utilizing multi-component seismic data.

It is emphasized that elastic and more complex modeling efforts come with a considerable additional cost. For example, the 3-D acoustic simulation performed in Figure 1 was performed in 10 hours on a typical desktop workstation (Sun Ultra 10), and required 300 MegaBytes of internal memory. The 3-D elastic simulation, however, required 14 hours of dedicated compute time using 240 CPU's and 80 GigaBytes of internal memory on a Compaq/DEC massively parallel computer. This was the largest seismic simulation performed in the world to date.

\section{Conclusions}

Traditional imaging and seismic modeling techniques assume that the earth can be described as a simple medium in an acoustic half-space. This assumption was used for the generation of the large SEG/EAGE acoustic numerical data set, which simulated 3-D seismic surveys in synthetic subsalt and overthrust geologic models. However, this acoustic assumption does not accurately depict important characteristics of seismic wave propagation that exist in realistic geologic environments. These characteristics arise from elastic mode converted energy, viscoelastic attenuation, anisotropy, and topography.

One of the most significant limitations of the acoustic assumption is the failure to model elastic wave propagation. Acoustic energy is partially converted into elastic energy at the free-surface and at boundaries within the geologic medium. The reverse is also true. This mode converted energy has two consequences for seismic modeling and imaging. The first consequence is "elastic noise", where an elastic signal is mistakenly interpreted as geologic structure. In addition, acoustic amplitudes are incorrectly modeled because some energy is lost to mode converted waves. The second consequence is that elastic modeling contains additional information about the subsurface geologic structure. It may be possible to incorporate this information into the seismic imaging process, and hence improve the ability to resolve complex geologic structures. These issues are further 


\section{Next-generation numerical modeling}

compounded by the influence of attenuation, anisotropy, and topography.

To address these shortfalls, a collaborative effort has been initiated between two Department of Energy national laboratories and the Society of Exploration Geophysicists to determine what characteristics and features the next generation of numerical seismic models will need to best address current technical problems in the processing and imaging of single and multi-component exploration data. This collaborative work is focused on designing and building these new models, generating synthetic seismic data through simulated surveys of various geometries, and using these data to test and validate new and improved seismic imaging algorithms. These models will include complex velocity structures, elastic medium parameters, viscoelastic attenuation, and anisotropy. Attention is being focused on multi-component data because it is now widely recognized that converted phases can play an important role in improving the quality of seismic images. Preliminary elastic modeling results demonstrate the importance of modeling seismic wave propagation in complex environments.

An existing, validated 3-D elastic modeling code is being used to generate the synthetic data. E3D is an explicit finitedifference code capable of simulating seismic wave propagation in large and complex geologic models. This code incorporates a number of advanced features including full 3-D elastic modeling, viscoelastic modeling, free-surface topography, propagating and variable density grids, hybridization, and parallelization. Anisotropic modeling capabilities are being implemented. The code runs on a number of platforms, from desktop workstations to high performance computers and massively parallel processors. This computing power is needed to simulate wave propagation in 3-D elastic and other physically complex models.

\section{Acknowledgments}

This work is supported by the U.S. Department of Energy, Office of Fossil Energy, through the Natural Gas and Oil Technology Partnership. In-kind and collaborative support for the model design effort is being provided by cost-sharing with industry partners and technical guidance from the SEG Research Committee. Computer time was made available through the Multi-programmatic and Institutional Computing program at Lawrence Livermore National Laboratory. This work was performed under the auspices of the U.S. Department of Energy by Lawrence Livermore National Laboratory under Contract W-7405-ENG-48, and by Los Alamos National Laboratory under Contract W-7405-ENG36.

\section{References}

Berger, M. J., and P. Colella, 1989, Local adaptive mesh refinement for shock hydrodynamics, J. Comp. Phys., 82, 6484.

Carcion, J. M., D. Kosloff, and R. Kosloff, 1988, Wave propagation simulation in an elastic anisotropic (transversely isotropic) solid, Q. J. Mech. Appl. Math., 41, 319-415.

Cerjan, C., D. Kosloff, R. Kosloff, and M. Reshef, 1984, A nonreflecting boundary condition for discrete acoustic and elastic wave equations, Geophysics, 50, 705-708.

Clayton, R., and B. Engquist, Absorbing boundary conditions for acoustic and elastic wave equations, Bull. Seism. Soc. Am., 67, 1529-1540.

Graves, R., 1996, Simulating seismic wave propagation in 3-D elastic media using staggered-grid finite differences, Bull. Seism. Soc. Am., 86, 1091-1106.

House, L., M. Fehler, F. Aminzadeh, J. Barhen, S. Larsen, 1996, A national laboratory-industry collaboration to use the SEG/EAEG model data sets, The Leading Edge, 15, 135-136.

Kessinger, W., and M. Ramaswamy, 1996, Subsalt imaging using mode converted energy and acoustic depth migration, Soc. Expl. Geophys., Expanded Abstracts, 66, 566-569.

Larsen, S., and J. Grieger, 1998, Elastic modeling initiative, Part III: 3-D computational modeling, Soc. Expl. Geophys. Confer. Proceed., 68, 1803-1806.

Levander, A. R., 1988, Fourth-order finite-difference P-SV seismograms, Geophysics, 53, 1425-1436.

Madariaga, R., 1976, Dynamics of an expanding circular fault, Bull. Seism. Soc. Am., 66, 639-666.

McLaughlin, K. L., and S. M. Day, 1994, 3D elastic finitedifference seismic-wave simulations, Computers in Physics, 8 , 656-663.

Mora, P., 1989, Modeling anisotropic seismic waves in 3-D, Soc. Expl. Geophys., Expanded Abstracts, 59, 1039-1043.

Ogilvie, J. S., and G. W. Purnell, 1996, Effects of salt-related mode conversions on subsalt prospecting, Geophysics, 61, 331-348.

Robertsson, J. O. A., J. O. Blanch, and W. W. Symes, 1994, Viscoelastic finite-difference modeling, Geophysics, 59, 14441456.

Schultz, C. A., 1997, A density-tapering approach for modeling the seismic response of free-surface topography, Geophys. Res. Lett., 24, 2809-2812.

Virieux, J., 1986, P-SV wave propagation in heterogeneous media: velocity-stress finite-difference method, Geophysics, 51, 889-901. 